\title{
Mucopolysaccharidosis VII: A case report and literature review.
}

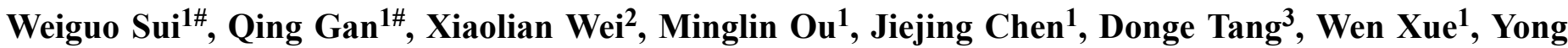 \\ Dai $^{1,3 *}$
}

${ }^{1}$ Nephrology Department of Guilin $181^{\text {st }}$ Hospital, Guangxi Key laboratory of Metabolic Diseases Research, Guilin, Guangxi, PR China

${ }^{2}$ College of Life Science, Guangxi Normal University, Guilin, Guangxi, PR China

${ }^{3}$ Clinical Medical Research Center, the Second Clinical Medical College of Jinan University (Shenzhen People's Hospital), Shenzhen, Guangdong, PR China

\#These authors contributed equally to this work

\begin{abstract}
Mucopolysaccharidosis VII (MPS VII) is a rare, genetic disease that is usually inherited as an autosomal-recessive trait. Genetic mutations affect mental retardation, skeletal deformities, corneal clouding, hepatosplenomegaly and intelligence. To date, several genes have been found in related to patients with MPS VII. Here, we used whole-exome sequencing to identify a mutation (NC_000007.13:g. $65444706 G>A)$ in $G U S B$ gene in an eight year old boy with MPS VII. Subsequently, we speculated that the variant of $G U S B$ gene is critical for the proper function of $G U S B$, and might had led to an absence of the $\beta$-glucuronidase or reduce activity of the enzyme.
\end{abstract}

Keywords: Mucopolysaccharidosis VII, Case report, Literature review, GUSB, Mutation.

Accepted on April 11, 2018

\section{Introduction}

Mucopolysaccharidosis VII (MPS VII) is a rare autosomal recessive condition classified in the group of mucopolysaccharide storage diseases [1]. It results from a deficient function of the enzyme $\beta$ glucuronidase (GUS: b-Dglucuronoside glucuronosohydrolase, EC 3.2.1.31; GUSB; MIM 611499) [2,3]. Deficiency of beta-glucuronidase (GUSB) results in progressive accumulation of undegraded glycosaminoglycans (GAGs) in various tissues including the brain $[3,4]$. The accumulation of glycosaminoglycansits may disrupt various processes of physiological and biochemical: the pathways related to gangliosides metabolism, receptormediated signaling, mechanisms related to the activation of inflammation, the intracellular and extracellular homeostasis of these macromolecules, oxidative stress and permeability of the lysosomal membrane, and alterations in intracellular ionic homeostasis and the endosomal pathway [5].

MPS VII was the first to be described in 1973 by Sly [6], and it is rare with the incidence rate $1 / 84000 \sim 1 / 130000$ [7]. As an autosomal recessive hereditary disease, Mucopolysaccharidosis type VII usually occurs on the offspring of the marriage between first Cousins. The patients' clinical manifestations are facial dysmorphism, skin coarse, diminutive, skeletal deformity, hepatosplenomegaly, retinal degeneration, stiff joints, cognitive deficits, hearing impairment and movement restricted $[7,8]$. At present, there is no special treatment for MPS VII. If we do not treat it, death will occur during adolescence. As a result, it poses a significant burden on society.

Humans [6], cats [9,10], dogs [11], and mice [12-14] have all been used as research models for MPS VII. Due to the complexity and severity of disease between MPS VII mice and Sly disease patients are similarity, MPS VII mice has been used extensively as a model system to study different therapeutic interventions [15]. According to the disease time and different degrees of severity, MPS VII can be classified as 3 sub-types. Subtypes I and subtypes II have severe phenotype and characteristics of early onset, manifests respectively on birth or within 4 years old. Their clinical performance are edema, multiple Skeletal malformations (dislocation of the hip joint contracture, thoracolumbar side after a sudden), giant deformity, coarse facial features, hepatosplenomegaly, inguinal hernia, navel hernia, recurrent upper respiratory tract infections, growth retardation, etc. Subtype III has mild manifestation and characteristics of late onset. Their clinical performances are mental retardation, and maculae corneae [16].

The 3 phenotypes appear to be caused primarily by different combinations of mutant alleles at the $\beta$-glucuronicacid Glycosides Enzyme Gene (GUSB). The GUSB gene that 
affects enzyme activity is located on chromosome 7q21 and 11-q22.1, which span approximately $20 \mathrm{~kb}$ and containing 11 introns and 12 exons. To date, 49 mutations, including 36 missense mutations, 6 non-sense mutations, 2 splice site mutations, and 5 deletions mutations in the GUS gene have been reported in MPS VII patients [7,17]. Some exonic point mutations, including p.L176F, p.R357X, p.P408S, p.P415L, and p.A619 V have been reported to be the most frequent mutations. Demonstration of the correlation of genotypes and phenotypes attempts to influence phenotypes by linking certain missense mutations or enzyme activity and stability [17]. Mutations in GUSB result in structural changes in the GUSB protein and loss of $\beta$ glucuronidase enzyme activity.

The mechanism of MPS VII is not fully clear. In this report, we described the mutations in the patient and his parents. We used the new technology of whole exome sequencing to study MPS VII of the skeleton with incomplete penetrance in different family members. And we hope this report will contribute to a better understanding of the genetic background in MPS VII patients. Further clinical, biochemical, and molecular studies are needed to establish better genotype-phenotype correlations in MPS VII mutations and symptoms.

\section{Case Report}

\section{Patient}

Clinical data was collected through the parents of patient interviews, medical records, and physical examinations. The patient with stunting, walking instability, mental retardation, forehead convex eyebrows thick, low nose, wide eyes, thick breathing, corneal slightly turbid, sluggish eyes, sleep snoring, mouth shape, weak vision, sparse teeth. The clinical details of the radiographically unaffected gene carriers and affected gene carriers in the MPS VII family were shown in Figure 1. The proband (IV: 5) of the MPS VII family, the son of a consanguineous parent, was a 7 year old boy. This boy was the first child of healthy parents. And he has a normal younger sister. He was born at term with normal length and weight. He was diagnosed with Mucopolysaccharidosis VII at the age of 7 years in Second Hospital Affiliated to Wenzhou Medical College. He and his parents came to Second Hospital Affiliated to Wenzhou Medical College on account of abnormal growth and development. His brain is dysplastic, with IQ number 11, extremely severe mental retardation on account of abnormal growth and development.

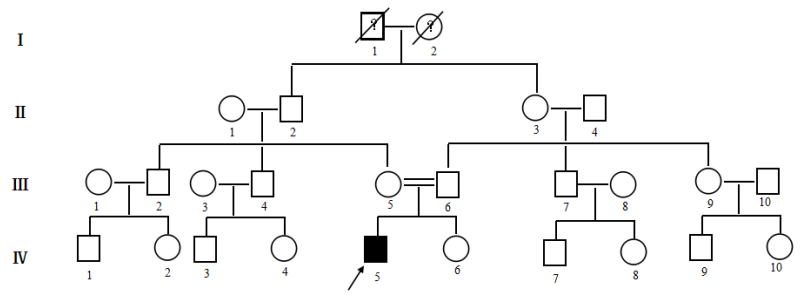

Figure 1. Pedigree of the MPS VII family included in the present study (arrows indicate the proband).

\section{Whole exome sequencing}

Whole-exome sequencing and analysis protocols was developed by the Human Genome Sequencing Center at the Baylor College of Medicine [18]. Briefly, genomic DNA samples were fragmented with the use of sonication, ligated to Illumina multiplexing paired-end adapters, amplified by means of a polymerase-chain-reaction assay with the use of primers with sequencing barcodes (indexes), and hybridized to biotinlabeled VCRome (version 2.1) [19]. The exomes of the patient (IV: 5) were captured and sequenced. The average sequence read depth was $81 \mathrm{X}$ in targeted exons. Sequencing data was aligned to the NCBI human reference genome (NCBI build 37) and compared to NCBI Single nucleotide variation detection (dbSNP) Build 132, 1000 Genomes Project, HapMap project and YH data-base. A total of 1912 genetic variations, including nonsynonymous mutations, splice-acceptor and splice-donor site variations, and Insertion deletion site detection (InDels), were identified from the individual (IV: 5) in the Mucopolysaccharidosis VII family.

In consideration that MPS VII is an inherited disease in family, variants in the individual were chosen for further analysis. Whole-genome exon sequencing results were screened out by the human gene bank dbSNP129, the international human genome haplotype map program (abbreviated HapMap program) exome data, and the exome data of 1000 genome projects. There were 1615 missense mutations and 297 deletions. Then, this study used ANNOVAR software to predict potentially harmful mutation sites to narrow down the candidate genes, used the GERP score to rank candidate genes for harmfulness, and focused on the genes which had been reported involving in Mucopolysaccharidosis VII of cats, dogs, mice and humans. The gene $\beta$-Glucuronidase $(G U S B)$ has been reported to be related to MPSVII. It was first suggested that the candidate gene GUSB (NC_000007.13: g.65444706G>A) may be related to MPSVII. The coverage in the exome sequencing of the variant in GUSB was $35 \mathrm{X}$ and 191X. Using traditional Sanger sequencing to verify, no candidate gene $G U S B$ (13: g. $65444706 \mathrm{G}>\mathrm{A})$ mutation site was found in other members of the family. In addition, the mutation (NC_000007.13:g. $65444706 \mathrm{G}>\mathrm{A}$ ) in $G U S B$ have not been well characterized, but mutations in GUSB were found in Mucopolysaccharidosis VII patients by other researchers, and we therefore supposed this variant as a pathogenic mutation of this family.

\section{Mutation analyses}

$\beta$-Glucuronidase (GUSB) is a lysosomal enzyme. It's important that GUSB in the normal step-wise degradation of glycosaminoglycans. GUSB is encoded by GUSB gene [20].

The $\beta$-glucuronidase is a lysosomal homotetramer [21], and comprised of 651 amino acid residues $(78 \mathrm{kD})$ [22]. The GUSB monomer is comprised of three distinct structural domains. The first domain is a jelly roll shape, which is highly distorted and forma a barrel-like structure along with two b-hairpin insertions. The hairpin loop of the jelly roll motif was considered to be an essential part of GUSB for lysosomal 
targeting. Therefore, it is also called a lysosomal targeting motif [23]. The second domain is structurally similar to the immunoglobulin constant domains. The third is ana/bor TIM barrel domain motif, which possesses the active site of the enzyme and a characteristic feature of glycosyl hydrolase enzymes $[24,25]$. The active site of each monomer is present at the interface of oligomer, and the tetramer complex has four active sites. Multiple sequence alignment of human GUSB with related sequences suggests that active-site residues are highly conserved in all glycosyl hydrolases [24,25]. This was a landmark discovery of the crystal structure of human GUSB in the area of lysosomal targeting [23]. This high-resolution crystal structure of GUSB provided a precise mechanism for lysosomal targeting [21].

The identified variant of GUSB (NC_000007.13:g. $65444706 \mathrm{G}>\mathrm{A}$ ) was a missense mutation in the proband. In the position 65444706 of chromosome $7, \mathrm{G}$ was substituted by A, which led to change of amino acid.

\section{Discussion}

Type VII mucopolysaccharides disease is known as Sly syndrome, which is the most recently recognized mucopolysaccharidosis associated with $\beta$-gluconidase deficiency [3]. The disease is caused by beta-D-glucuronidase deficiency. Deficiency of GUSB causes partial degradation of Heparan Sulfate (HS), Chondroitin Sulfate (CS), Dermatan Sulfate (DS). Degradants accumulate in the lysosomes of many tissues, which lead to cell damage and organ dysfunction $[23,26]$.

Mucopolysaccharide VII is a disease caused by accumulation of GAGs due to lysosomal defects, the incidence of a disease is 1/840000-1300000 [7]. In China, the first MPS VII was reported in 2011 by Huang et al [7]. They found that the patient with MPS type VII was a 2 months baby boy. The boy was mainly characterized by edema, liver splenomegaly, oblique hernia and decreasing platelets. The urine excretion of patient significantly increased, and patient finally was definited diagnosis by the leukocytes of peripheral blood and activity of $\beta$-glucuronic acid glycosides enzyme. Then the patient gradually appeared severe edema, psychomotor retardation and rough appearance. The leukocytes of peripheral blood were bulky. Alder-Reilly can be seen in the cytoplasm. The patient was dead after 6 months.

Mucopolysaccharide VII disease is caused by a loss of lysosomal hydrolase activity due to a mutation in the $\beta$ glucuronidase gene. Mutation of $\beta$-glucuronidase gene (GUSB MIM *611499) have been described in many species including human, mouse, cat and dog [7,21]. To this date, forty-nine unique disease-causing mutations (missense mutations, deletions, nonsense mutations, splice-site mutations) in GUSB have been identified in person with MPS VII from various populations. Among these mutations, L176F is one of the most commonly reported mutations in MPSVII patients [22]. Benjamin et al. [27] first identified the L176F (Leu $\rightarrow$ Phe) of mutation in exon 3 of the beta-glucuronidase gene in mennonite siblings with beta-glucuronidase-deficien. The mutant enzyme was proved to be much more labile than the wild- type enzyme to inactivity on by incubation at $65^{\circ} \mathrm{C}$ (labile can cause a remarkable decrease in the enzyme activity). They identified the level of expression of the L176F mutation in many tissues of the Mennonite siblings, which was low enough to produce the MPS VI1 phenotype.

Vervoort et al. [28] identified a pseudo deficiency allele (Asp152Asn) of GUSB and observed a reduced protein, stability that causes a remarkable decrease in the enzyme activity. Another mutation reported in the early infantile MPSVII was Tyr626His, which was localized in the lasta-helix of the TIM barrel and is in close proximity to two mutations (Ala619Val and Trp627Cys). Such a mutation affects hydrophobic core packing of GUSB.

Yamada et al. [29] reported several novel mutations, Pro148Ser, Tyr495Cys, Trp507X, and a 38-bp deletion at position 1642-1679 in exon 10 (1642D38nt) of MPSVII patients. Among these, the truncating mutation of Trp507X produces a more severe MPSVII phenotype. It is interesting to note that no mutation in active-site residues Glu540, Glu451, or Tyr504 was reported in MPSVII patients. Such active-site mutations would possibly produce severe clinical consequences, which render patients incompatible to survive. A comprehensive analysis of structural changes with respect to mutation sites have already been reported earlier [30,31].

Gitzelmann et al. [32] found a pathogenic mutation (c. $155 \mathrm{C}>\mathrm{T}$ ) only in their case of MPS VII. The c. $155 \mathrm{C}>\mathrm{T}$ mutation was located in exon 1 . And it was predicted that the phenotypic severity in MPS VII patient was predominantly influenced by the c. $155 \mathrm{C}>\mathrm{T}$ mutation, which caused total destruction of $\beta$-glucuronidase enzyme activity in transfected cells. In addition, another mutation (c.1120C $>\mathrm{T}$ ) had been further detected in one allele of a German proband before [33]. The c.1120C $>$ T mutation might have led to an absence of the mental retardation because the $\mathrm{c} .1120 \mathrm{C}>\mathrm{T}$ mutation led to $\beta$ glucuronidase a residual activity of $5.5 \%$ compared with the wild type [34].

In our case, the mutation which we hadn't found in previous studies was heterozygotes. The mutation (G was substituted by A) had led to threonine change into serine. And we speculated that the variant of GUSB (NC_000007.13:g.65444706G $>$ A) was critical for the proper function of GUSB, and might had led to an absence of the $\beta$-glucuronidase or reduce activity of the enzyme.

In summary, mucopolysaccharidos VII as a very rare disease closely related to genetic factors. At present, foreign scholars have reported the related genes of the disease, but no further molecular genetic studies have been conducted in China. We used whole exome sequencing to identify the pathogenic gene disease in the MPS VII patient, and rapidly identify NC_000007.13:g.65444706G $>$ A of a point mutation in GUSB of patient. The study of the pathogenic mutations will provide theoretical basis for revealing the molecular genetic features of the disease and the prenatal diagnosis and treatment. Whole 
genome sequencing is used as a supplementary method for the diagnosis of mucopolysaccharidosis VII, and at the same time it will be helpful for the targeted treatment of mucopolysaccharidosis VII.

\section{Funding}

This work was supported by Science and Technology Planning Project of Guangdong Province, China (No. 2017B020209001), Project Plan Document of Guangxi Key Laboratory Construction (17-259-57).

\section{References}

1. Tomatsu S, Montano AM, Dung VC. Mutations and polymorphisms in GUSB gene in mucopolysaccharidosis VII (Sly Syndrome). Human Mut 2009; 30: 511-519.

2. Neufeld EF, Muenzer J. The meratabolic anad neoldnelar basis of inherited dtncase. Vcw York: McGraw Hill 1995; 2466-2494.

3. Sly WS, Quinton BA, McAlister WH, Rimoin DL. Beta glucuronidase deficiency: report of clinical, radiologic, and biochemical features of a new mucopolysaccharidosis. J Pediatr 1973; 82: 249-257.

4. Neufeld EF, Muenzer J. The mucopolysaccharidosis. Metabolic Basis of Inherited Disease. McGraw-Hill Inc. New York 1989; 3421-3452.

5. Derbis C, Madelyn M. Mucopolysaccharidosis type I: current knowledge on its pathophysiological mechanisms. Metab Brain Dis 2012; 27: 121-129.

6. Sly WS, Quinton BA, McAlister WJ, Rimoin DL. Betaglucuronidase deficiency: report of clinical, radiologic and biochemical features of a new mucopolysaccharidosis. J Pediatr 1973; 82: 249-257.

7. Huang Y, Li S, Zhao X. Mucopolysaccharidosis VII: report of a case and renew of the literature. Chin J Pediatr 2011; 49: 455-460.

8. Donsante L, Vogler SMS. Clinical response to persistent, low-level $\beta$-glucuronidase expression in the murine model of mucopolysaccharidosis type VII. J Inherit Metab Dis 2007; 30: 227-238.

9. Fyfe JC, Kurzhals RL, Lassaline ME. The molecular basis of felineb-glucuronidase deficiency: an animal model of mucopolysaccharidosis VII. Genomics 1999; 58: 121-128.

10. Gregory GH, Annette FSRK. Accumulation of abnormal amounts of glycosaminoglycans in murine mucopolysaccharidosis type VII neural progenitor cells does not alter the growth rate or efficiency of differentiation into neurons. Mol Cell Neurosci 2001; 17 : 167-178.

11. Haskins ME, Desnick RJ, Diferrante N, Jezyk PF, Patterson DF. Beta-glucuronidase deficiency in a dog: a model of mucopolysaccharidosis VII. Pediatr Res 1984; 18: 980-984.

12. Birkenmeier EH, Davisson MT, Beamer WG. Murine mucopolysaccharidosis type VII: characterization of a mouse withb-glucuronidase deficiency. J Clin Invest 1989; 83: 1258-1266.

13. Gwynn B, Lueders K, Sands MS, Birkenmeier EH. Intracisternal A-particle element transposition into the murine b-glucuronidase gene correlates with loss of enzyme activity: a new model for b-glucuronidase deficiency in the $\mathrm{C} 3 \mathrm{H}$ mouse. Mol Cell Biol 1998; 18: 6474-6481.

14. Casal ML, Wolfe JH. Variant clinical course of mucopolysaccharidosis type VII in two groups of mice carrying the same mutation. Lab Invest 1998; 75: 1575-1581.

15. Gitzelmann R, Bosshard NU, Supertifurga A. Feline mucopolysaccharidosis-VII due to betaglucuronidase deficiency. Vet Pathol 1994; 31: 435-443.

16. Lee J, Falk R, Ng W. Beta-glucuronidase deficiency: a heterogenous mucopolysaccharidosis. Am J Dis Child 1985; 139: 57-59.

17. Tomatsu S, Montario AM, Dung VC. Mutations and polymorphisms in GUSB gene in mucopolysaccharidosis VII (Sly Syndrome). Hum Mutat 2009; 30: 511-519.

18. Yaping Y, Donna MM, Jeffrey G. Clinical whole-exome sequencing for the diagnosis of mendelian disorders. $\mathrm{N}$ Engl J Med 2013; 369: 1502-1511.

19. Bainbridge MN, Wang M, Wu Y. Targeted enrichment beyond the consensus coding DNA sequence exome reveals exons with higher variant densities. Genome Biol 2011; 12: 68 .

20. Carole V, Nancy G, Beth L. Transgene produces massive overexpression of human $\beta$-glucuronidase in mice, lysosomal storage of enzyme, and strain-dependent tumors. Proc Natl Acad Sci USA 2003; 100: 2669-2673.

21. Hytonen MK, Arumilli M, Lappalainen AK. A novel GUSB mutation in Brazilian terriers with severe skeletal abnormalities defines the disease as mucopolysaccharidosis VII. PLoS One 2012; 7: 1-11.

22. Huma N, Asimul I, Abdul W. Humanb-glucuronidase: structure, function, and application in enzyme replacement therapy. Rejuv Res 2013; 5: 352-363.

23. Jain S, Drendel WB, Chen ZW. Structure of human betaglucuronidase reveals candidate lysosomal targeting and active-site motifs. Nat Struct Biol 1996; 3: 375-381.

24. Sinnott ML. Ions, ion-pairs and catalysis by the lacZ betagalactosidase of Escherichia coli. FEBS Lett 1978; 94: $1-9$.

25. Gebler JC, Aebersold R, Withers SG. Glu-537, not Glu-461, is the nucleophile in the active site of (lac Z) betagalactosidase from Escherichia coli. J Biol Chem 1992; 267: 11126-11130.

26. Delbecque K, Gaillez S, Schaaps JP. Histopathological diagnosis of a type vii mucopolysaccharidosis after pregnancy termination. Fetal Pediatr Pathol 2009; 28: 1-8.

27. Benjamin MWH, Shuqji T, Seiji F. Overexpression rescues the mutant phenotype of L176F mutation causing $\beta$-glucuronidase deficiency mucopolysaccharidosis in two 
mennonite siblings. J Biol Chem 1994; 269: 23681-23688.

28. Vervoort R, Islam MR, Sly W, Chabas A, Wevers R, de Jong J, Liebaers I, Lissens W. A pseudodeficiency allele (D152N) of the human beta-glucuronidase gene. Am J Hum Genet 1995; 57: 798-804.

29. Yamada S, Tomatsu S, Sly WS, Islam R, Wenger DA, Fukuda S, Sukegawa K, Orii T. Four novel mutations in mucopolysaccharidosis type VII including a unique base substitution in exon 10 of the beta-glucuronidase gene that creates a novel $5 \phi$-splice site. Hum Mol Genet 1995; 4: 651-655.

30. Islam MR, Tomatsu S, Shah GN, Grubb JH, Jain S, Sly WS. Active site residues of human beta-glucuronidase. Evidence for Glu (540) as the nucleophile and Glu (451) as the acid-base residue. J Biol Chem 1999; 274: 23451-23455.

31. Tomatsu S, Montano AM, Dung VC, Grubb JH, Sly WS. Mutations and polymorphisms in GUSB gene in mucopolysaccharidosis VII (Sly Syndrome). Hum Mutat 2009; 30: 511-519.

32. Robert DY, Petra L, Christian P. Large proteoglycan complexes and disturbed collagen architecture in the corneal extracellular matrix of mucopolysaccharidosis type VII (Sly syndrome). Investigative Ophthalmol Vis Sci 2011; 52: 6720-6728.

33. Vervoort R, Islam MR, Sly WS. Molecular analysis of patients with -glucuronidase deficiency presenting as hydrops fetalis or as early mucopolysaccharidosis VII. Am J Hum Genet 1996; 58: 457-471.

34. Gitzelmann R, Wiesmann UN, Spycher MA, Herschkowitz N, Giedion A. Unusually mild course of Bglucuronidase deficiency in two brothers (mucopolysaccharidosis VII). Helv Pediatr Acta 1978; 33: 413- 428

\section{*Correspondence to}

Yong Dai

Nephrology Department of Guilin $181^{\text {st }}$ Hospital

Guangxi Key laboratory of Metabolic Diseases Research

PR China 